\title{
A rare presentation of adrenal adenoma in infancy: isolated Cushing's syndrome
}

\author{
Oğuzhan Şal ${ }^{1 \oplus}$, Gülçin Yeğen², Pelin Oğuzkurt ${ }^{3}$ \\ ${ }^{3}$ Department of Pediatric Surgery, ${ }^{1}$ Koç University School of Medicine, İstanbul; ${ }^{2}$ Department of Pathology, İstanbul University \\ Istanbul Faculty of Medicine, İstanbul, Turkey.
}

\begin{abstract}
Adrenocortical tumors are rare in children. Most of these tumors present with endocrinological manifestations, majority of which with virilizing features alone or in combination with over production of other adrenal hormones. However, it is uncommon of an adrenocortical tumor to present solely with Cushing's syndrome. In this paper we discuss the clinical presentation and management of a 5-month-old infant who had presented with Cushing's syndrome due to a functioning adrenocortical adenoma without androgen and mineralocorticoid excess, and made a brief review on the clinical and histopathological characteristics of adrenocortical tumors.
\end{abstract}

Key words: adrenocortical, adenoma, Cushing's syndrome, non-virilizing, wieneke.

Adrenocortical tumors (ACT) are among the rarest malignancies in children. ${ }^{1}$ The annual worldwide incidence of childhood ACT is reported to be $0.3-0.38$ per million children below the age of 15 years. The term ACT in children includes both benign adrenocortical adenomas (ACA) and malignant adrenocortical carcinomas (ACC), however histopathological classification of ACT is troublesome. Adrenocortical carcinomas are associated with recurrences and furthermore with death with a 5-year event free survival estimate of 46$54 \% .^{2}$ Best prognostic factors for ACT are early detection, complete resection of the mass, and good constructed pre and post-operative steroid regimen. ${ }^{3}$ It is already known that most of the ACT are endocrinologically active and usually present with virilization, precocious puberty with or without Cushing syndrome (CS) and rarely with isolated CS. ${ }^{1,4}$

The differentiation of malignant from benign tumor is difficult even with histopathological examination; therefore, final diagnosis relies

$凶$ Oğuzhan Şal

osal13@ku.edu.tr

Received 20th November 2018; revised 30th January 2019, 27th February 2019, 4th April 2019; accepted 24th April 2019 on the evaluation of clinical manifestations, laboratory data, imaging, histopathological evaluation and clinical behavior of the tumor. Wieneke Scoring System is used to distinguish between malignant and benign adrenal tumors. In this paper, we report an infant who presented with isolated CS related to right adrenal adenoma according to Wieneke criteria.

\section{Case Report}

A 5-month-old female infant was admitted to the pediatric clinic due to progressive weight gain and facial swelling. She was born to nonconsanguineous parents at the $38^{\text {th }}$ week of gestation by $\mathrm{C}$-section. Her birth weight was 3120 gr (25 percentile), birth height and head circumference were in 50 and between 25-50 percentiles, respectively. She was breastfed and later supplemented with formula feeding.

Her weight at presentation was $9 \mathrm{~kg}\left(>95^{\text {th }}\right.$ percentile). Vital signs including heart rate, blood pressure and respiratory rate were within normal limits for her age. In physical examination she had Cushingoid facies, buffalo hump, generalized excessive body fat, rashes in perianal and neck region. There were no signs of facial acne or virilization and she had 
no further remarkable physical examination findings. In laboratory examinations, serum biochemical laboratory results were within normal ranges Na:147nmol/L (normal:135-150), $\mathrm{K}: \quad 4.7 \mathrm{mmol} / \mathrm{L}$ (normal:3.5-5.5), urea:13md/ $\mathrm{dL} \quad$ (normal:11-38), creatinine: $0.4 \mathrm{mg} / \mathrm{dL}$ (normal:0.17-0.42), whereas morning cortisol was $939.1 \mathrm{nmol} / \mathrm{L}$ (normal: 111-656 nmol/L); Adrenocorticotropic hormone (ACTH) was less than 5pg/mL (normal: 10.0-63.3 pg/mL). Abdominal ultrasound showed a hypoechoic, well-defined solid mass of $3.5 \times 5 \mathrm{~cm}$, in the right adrenal region. Abdominal magnetic resonance imaging (MRI) showed regular,

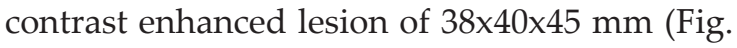
1a) which compressed the inferior vena cava (Fig. 1b). A perioperative premedication with intravenous methylprednisolone was given to prevent adrenal insufficiency.

Right adrenalectomy was performed through a right subcostal incision. The cranial portion of the lesion had invaded the Glisson's capsule of the liver and also compressed the inferior vena cava medially. The mass was completely excised with the adherent liver parenchyma.

Gross pathological examination revealed a solid, yellowish tumor adjacent to the liver parenchyma. It was measured $5 \times 4 \times 4 \mathrm{~cm}$ and weighed $35 \mathrm{~g}$. At microscopic examination encapsulated nodular lesion with trabecularfocally pseudoacinar pattern was seen (Fig. 2a). Tumor was consisted of eosinophilic cells with oval to round nucleus with fine chromatin. Although the tumor had expansive borders and was generally encapsulated, focally the capsule was wiped up and the tumor cells intermingled with the hepatocytes (Fig. 2b). There was no necrosis or vascular invasion. Four mitoses were observed at $20 \mathrm{HPF}$, but no atypical mitotic figures were observed. Immunohistochemically tumor cells were positive for melan-A (Fig. 2c), focally for inhibin alpha and were negative for cytokeratin, chromogranin A, synaptophysin, HepPar-1, glypican 3 and p53. Ki67 proliferation index was 10\% (Fig 2d). Histopathological findings were compatible with adrenocortical adenoma according to Wieneke classification system (Table I) and a Stage 1 adrenocortical tumor according to pediatric adrenocortical tumor staging criteria.

The patient showed an uneventful recovery and discharged with oral hydrocortisone 5 days after the surgery. At 3 months follow-up, oral hydrocortisone was tapered and stopped

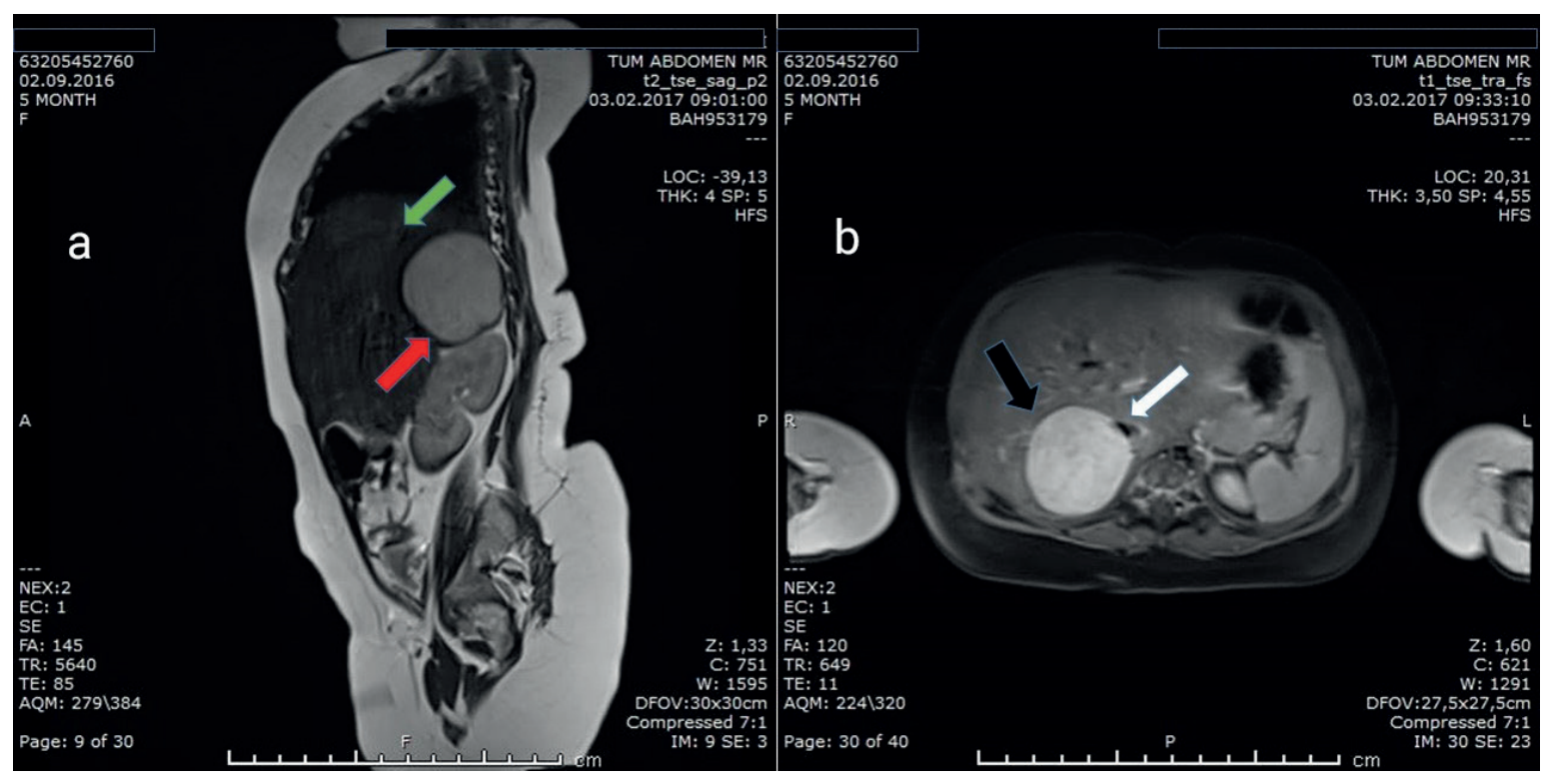

Fig. 1a. Right adrenal mass (shown with red arrow) and its close proximity to liver (shown with green arrow). 1b. The mass (shown with black arrow) and its compression on inferior vena cava (shown with white arrow). 


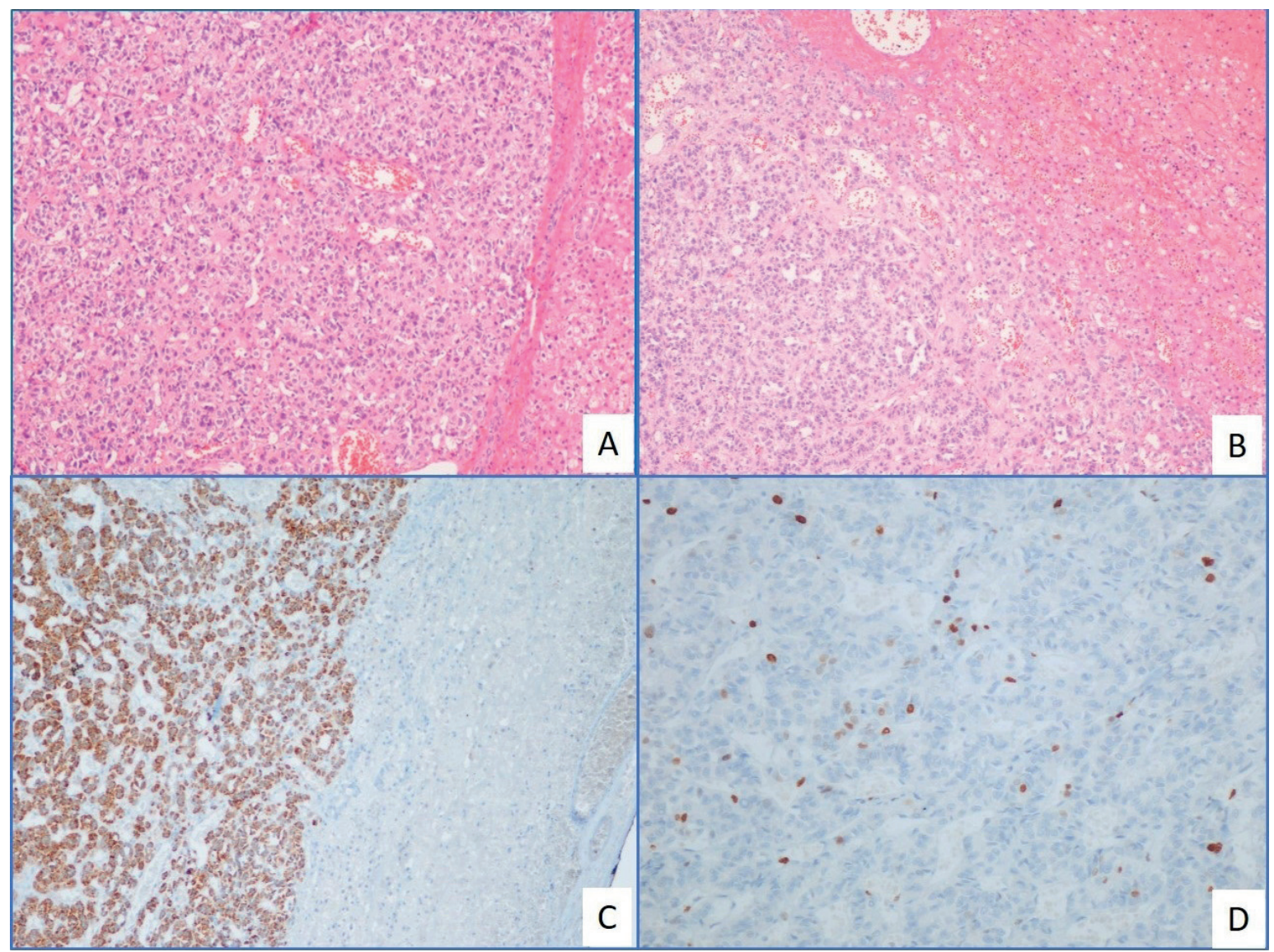

Fig. 2. Pathology sections of the tumor.

Table I. Wieneke criteria.

Wieneke criteria
Tumor weight $>400 \mathrm{gr}$
Tumor size $>10,5$
Extension into periadrenal soft tissues and/or adjacent organs*
Invasion into vena cava
Venous invasion
Capsular invasion*
Presence of tumor necrosis
$>15$ mitoses per $20 \mathrm{HPF}$
Presence of atypical mitotic figures

(*Positive criteria present in our case)

Tumors showing $\leq 2$ of features were classified as benign, $\geq 4$ criteria as malignant, whereas tumors with 3 features were classified as indeterminate for malignancy.

through regular ACTH and cortisol monitoring (Table II). The cortisol and ACTH levels were $4.3 \mu \mathrm{g} / \mathrm{dl}$ (normal range: $3.7-9.4 \mu \mathrm{g} / \mathrm{dl}$ ) and $17 \mathrm{pg} /$ $\mathrm{ml}$ (normal range:10-60 pg/ml), respectively at the time of cessation of replacement treatment.
The postoperative serum biochemistry results were normal. During the follow-up period, her Cushingoid appearance resolved at one-year of age (Fig $3 a$ and $b$ ). 


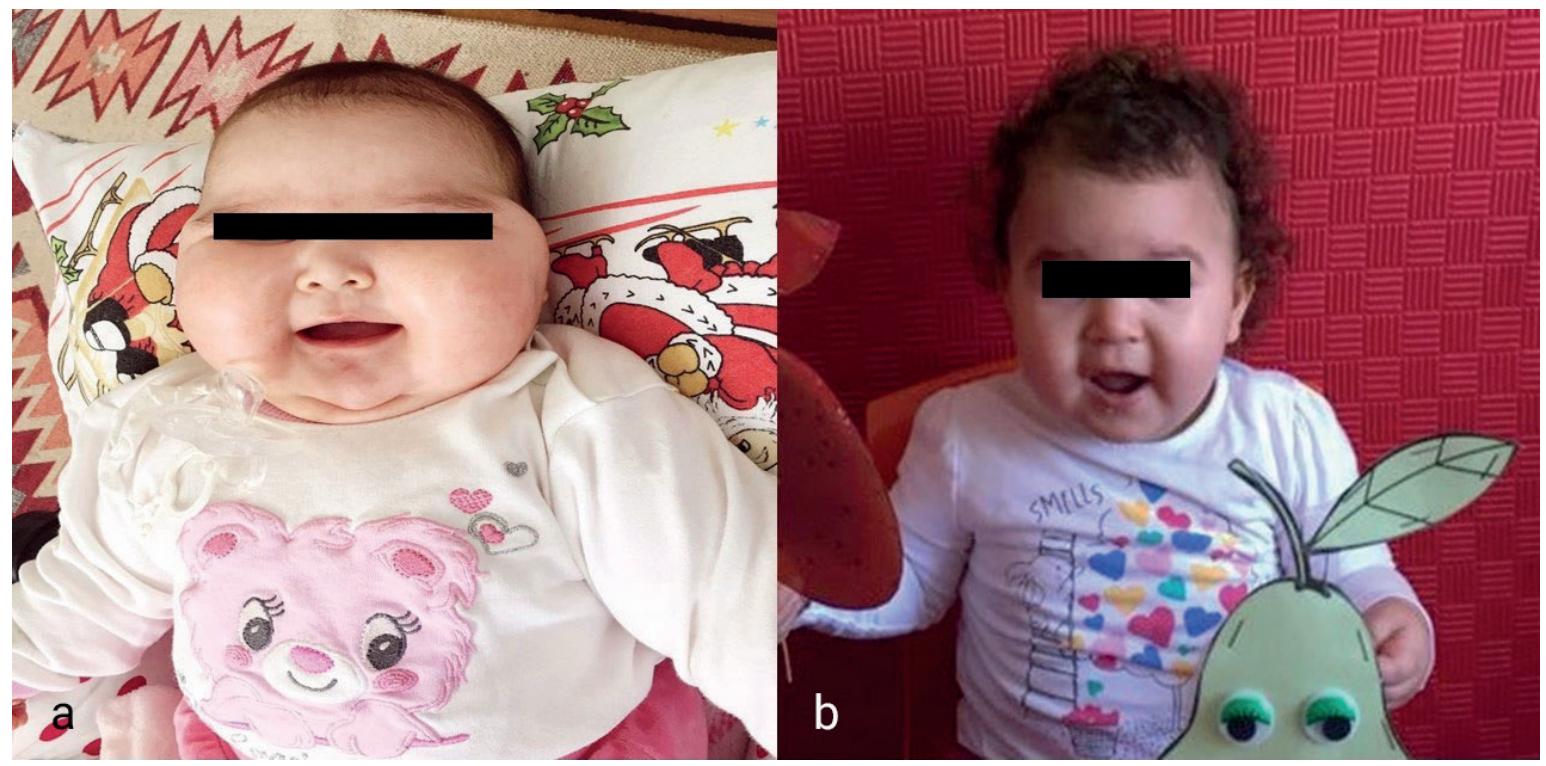

Fig. 3a and $\mathbf{b}$. Cushingoid facies of the patient before and after the treatment.

Table II. ACTH and cortisol levels of the patient before and after surgery.

\begin{tabular}{|c|c|c|}
\hline Date & $\mathrm{ACTH}$ & Cortisol \\
\hline 28.01 .2017 & $<5.00$ pg/mL (normal:10-63) & 939,1 nmol/L(normal:111-656) \\
\hline 08.02.2017 & \multicolumn{2}{|c|}{ Adrenalectomy was performed. } \\
\hline 15.02.2017 & 22,2 pg/mL (normal:10-63) & 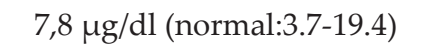 \\
\hline 03.03.2017 & 28,1 pg/mL (normal:10-63) & 4.0 g/dl (normal:3.7-19.4) \\
\hline 05.04.2017 & 31,8 pg/mL (normal:10-63) & 3.0 g/dl (normal:3.7-19.4) \\
\hline \multicolumn{3}{|c|}{ Cessation of replacement treatment } \\
\hline 02.05 .2017 & $17 \mathrm{pg} / \mathrm{mL}$ (normal:10-63) & 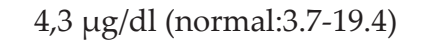 \\
\hline 02.06.2017 & 24 pg/mL (normal:10-63) & 8,7 g/dl (normal:3.7-19.4) \\
\hline 16.08.2017 & 14 pg/mL (normal:10-63) & 5,4 g/dl (normal:3.7-19.4) \\
\hline
\end{tabular}

An informed consent to publish the case report including the photos was obtained from parents of the patient.

\section{Discussion}

In this report we presented a 5-month-old girl who showed discriminating clinical features of glucocorticoid excess, particularly excessive weight gain, Cushingoid facies, buffalo hump, and generalized increased body fat. She had biochemical evidence of excessive glucocorticoid production and ACTH suppression. CS in pediatric population usually manifests by slowed linear growth, extremely accelerated weight gain, facial puffiness, buffalo hump; rarely it presents itself by virilizing symptoms such as pubic and axillary hair development, and breast development. ${ }^{5}$ CS can be ACTHdependent or ACTH-independent. ACTH independent causes are primary adrenocortical hyperfunction, adrenocortical tumors (ACT), primary pigmented nodular adrenocortical disease, bilateral macronodular adrenal hyperplasia, and iatrogenic CS. ${ }^{6}$

The serum cortisol level of the presented patient was elevated whereas the ACTH level was severely suppressed, therefore an ACTHindependent cause of CS was suggested to be the etiology after excluding the iatrogenic CS. Oral and topical glucocorticoid therapies 
in infants and young children with diaper dermatitis are the most common causes of iatrogenic CS, although other applications such as inhalation, ocular and nasal drops including glucocorticoids may also result in hypercortisolism. ${ }^{7}$ In iatrogenic CS, all affected cases have low ACTH and cortisol levels. The presented patient did not have a history of exposure to exogenous glucocorticoids.

Adrenocortical tumors are rare in infancy and occur primarily in children between one to five years of age $(60 \%)$, with a peak in incidence below 4 years of age. ${ }^{7}$ To the best of our knowledge ACA and ACC presenting early in infancy with isolated CS are very rare and reported through a few case reports. ${ }^{8-10}$

In approximately $15-20 \%$ of CS cases, ACT are the etiology behind the disease. After clinical and laboratory assessment; preferred radiological approaches are ultrasound, computerized tomography (CT) or magnetic resonance imaging (MRI) ; in MRI fat-rich nature of ACA is suggested to be quite useful in distinguishing them from ACC.$^{11,12}$ Although CT has been the primary choice of imaging, MRI has superior traits such as better visualization of possible anatomical relationship between the mass and the adjacent anatomical structures. ${ }^{11}$ FDG-PET scans are also quite useful in differentiation between ACC and ACA. ${ }^{13}$ In our patient abdominal ultrasonography clearly defined hypoechoic right adrenal tumor, which was confirmed with MRI as a contrast enhanced lesion compressing the inferior vena cava. Imaging studies help in the surgical planning and the staging of the disease as well as predicting the resectability of the lesion such as tumor size, invasion to the adjacent structures and vascular invasion or thrombosis. ${ }^{14}$

Surgery is the most important procedure in the treatment of ACT. Endocrinological preparation of the patient prior to operation is vital in order to successfully avoid life threatening acute adrenal insufficiency due to the hypoplastic contralateral adrenal gland secondary to prolonged suppression of ACTH secretion from the pituitary. ${ }^{15,16}$

Adrenocortical tumors in children have a less aggressive clinical behavior when compared to their adult counterpart and furthermore application of histopathological criteria of adult ACT will result mostly in overdiagnosis of pediatric cases as carcinoma. ${ }^{17-20}$ In childhood $\mathrm{ACT}$, there is no single pathological feature that can predict the malignant behavior. Wieneke et.al proposed a set of macroscopic and microscopic criteria for histopathologic diagnosis of malignant ACT. ${ }^{18}$ Nevertheless, the predictive value of the system is yet to be validated..$^{18}$ Besides the histopathologic criteria, a modified pediatric staging system based on tumor weight or volume and resectability was accepted to show prognostic significance. ${ }^{22}$ In our case, adverse pathological features that had been mentioned in Wieneke Scoring System were focal capsule invasion with subsequent liver parenchymal invasion. The presented patient was diagnosed as ACA with young age at presentation, small tumor size and low tumor volume, and absence of tumor necrosis and atypical mitotic figures; however, focal invasion of liver parenchyma was considered as an important factor in favor of malignancy, so a close follow-up with endocrinological evaluation and ultrasound examination is recommended.

Adrenocortical tumors are rare in pediatric population. They rarely represent themselves with isolated CS; in this paper we presented a rare clinical picture of ACA; a 5-month-old infant with an isolated CS associated with a right ACA. Pathophysiology, diagnostic laboratory tests, imaging findings and treatment modalities are discussed. According to the Wieneke criteria this case was diagnosed as an adenoma. Distinction between ACA and ACC is quite challenging, therefore further pathological clarification between them is significant, creating necessity of ACT registry to obtain large data to have new guideline criteria. 


\section{REFERENCES}

1. Sutter JA, Grimberg A. Adrenocortical tumors and hyperplasias in childhood - etiology, genetics, clinical presentation and therapy. Pediatr Endocrinol Rev 2006; 4: 32-39.

2. Xu X, Sergi C. Pediatric adrenal cortical carcinomas: histopathological criteria and clinical trials. A systematic review. Contemp Clin Trials 2016; 50: 3744 .

3. Lalli E, Figueiredo BC. Pediatric adrenocortical tumors: what they can tell us on adrenal development and comparison with adult adrenal tumors. Front Endocrinol (Lausanne) 2015; 6: 23.

4. Michalkiewicz E, Sandrini R, Figueiredo B, et al. Clinical and outcome characteristics of children with adrenocortical tumors: a report from the International Pediatric Adrenocortical Tumor Registry. J Clin Oncol 2004; 22: 838-845.

5. Lodish M, Keil MF, Stratakis CA. Cushing syndrome in childhood. In: Radovick S, Misra M. (eds). Pediatric Endocrinology: A Practical Clinical Guide. (3th ed). Switzerland: Springer, 2018: 335-353.

6. Richmond EJ, Rogol AD. Cushing's Disease in Children and Adolescents: Diagnosis and Management. Cushing's Disease: Elsevier BV, 2017: 187-200.

7. Çömlek FÖ, Örüm $S$, Aydın $S$, Tütüncüler F. Exogenous Cushing syndrome due to misuse of potent topical steroid. Pediatr Dermatol 2018; 35: e121-e123.

8. Dutta D, Jain R, Maisnam I, et al. Isolated Cushing's syndrome in early infancy due to left adrenal adenoma: an unusual aetiology. J Clin Res Pediatr Endocrinol 2012; 4: 164-168.

9. Fudge EB, von Allmen D, Volmar KE, Calikoglu A. Cushing syndrome in a 6-month-old infant due to adrenocortical tumor. Int J Pediat Endocrinol 2009; 2009: 168749.

10. Kim E, Aguayo P, St Peter SS, Holcomb 3rd GW. Adrenocortical adenoma expressing glucocorticoid in an 8-month-old female. Eur J Pediatr Surg 2009; 19: $265-267$.
11. Sargar KM, Khanna G, Hulett Bowling R. Imaging of nonmalignant adrenal lesions in children. Radiographics 2017; 37: 1648-1664.

12. Lodish MB, Keil MF, Stratakis CA. Cushing's syndrome in pediatrics. Endocrinol Metab Clin North Am 2018; 47: 451-462.

13. Guerin C, Pattou F, Brunaud L, et al. Performance of 18F-FDG PET/CT in the characterization of adrenal masses in noncancer patients: a prospective study. J Clin Endocrinol Metab 2017; 102: 2465-2472.

14. León MIM, Chaparro SR, Lara BW, et al. Adrenocortical tumors in children: imaging adenomas and carcinomas. Radiología (English Edition) 2012; 54: 342-349.

15. Stratakis CA. An update on Cushing syndrome in pediatrics. Ann Endocrinol (Paris) 2018; 79: 125-131.

16. Gow KW. The evaluation and management of adrenal masses and adrenocortical tumors (Act). In: Ledbetter DJ, Johnson PRV (eds). Endocrine Surgery in Children. Verlag GmbH Germany: Springer, 2018: 121-139.

17. Das $S$, Sengupta $M$, Islam N, et al. Weineke criteria, Ki-67 index and p53 status to study pediatric adrenocortical tumors: is there a correlation? J Pediatr Surg 2016; 51: 1795-1800.

18. Dehner LP, Hill DA. Adrenal cortical neoplasms in children: why so many carcinomas and yet so many survivors? Pediatr Dev Pathol 2009; 12: 284-291.

19. Weiss LM. Comparative histologic study of 43 metastasizing and nonmetastasizing adrenocortical tumors. Am J Surg Pathol 1984; 8: 163-169.

20. Weiss LM, Medeiros LJ, Vickery AL Jr. Pathologic features of prognostic significance in adrenocortical carcinoma. Am J Surg Pathol 1989; 13: 202-206.

21. Pinto EM, Rodriguez-Galindo C, Choi JK, et al. Prognostic significance of major histocompatibility complex class II expression in pediatric adrenocortical tumors: a St. Jude and Children's Oncology Group Study. Clin Cancer Res 2016; 22: 6247-6255.

22. Stewart JN, Flageole H, Kavan P. A surgical approach to adrenocortical tumors in children: the mainstay of treatment. J Pediatr Surg 2004; 39: 759763. 